\title{
Implementación de un programa de optimización de antibióticos en la unidad de cuidados intensivos de un hospital público de Santa Elena
}

Implementation of an antibiotic stewardship programme in the intensive care unit of a public hospital in Santa Elena

\author{
Pablo Ruales Carrion \\ Walter Mariscal Santi \\ William Jimenez Jimenez
}

Fecha de recepción:1 de mayo del. 2021.

Fecha de aceptación:23 de junio del. 2021 


\title{
Implementación de un programa de optimización de antibióticos en la unidad de cuidados intensivos de un hospital público de Santa Elena
}

\author{
Implementation of an antibiotic stewardship programme in the intensive care \\ unit of a public hospital in Santa Elena \\ Pablo Ruales Carrion ${ }^{1}$. Walter Mariscal Santi ${ }^{2}$. William Jimenez Jimenez ${ }^{3}$.
}

Como citar: Ruales, P., Mariscal, W., Jimenez, W., (2021). Implementación de un programa de optimización de antibióticos en la unidad de cuidados intensivos de un hospital público de Santa Elena, Revista Universidad de Guayaquil. 132(2), 9-16. DOI: https://doi.org/10.53591/rug.v133i2.1379

\section{RESUMEN}

La implementación de los Programas de Optimización de Antibióticos (PROA) han demostrado ser herramientas eficaces para la seguridad de los pacientes a través de un uso adecuado de los antibióticos por medio varias determinaciones microbiológicas de resistencia de los microorganismos a los antibióticos seleccionados para este estudio. En el presente trabajo se realizó la implementación de un PROA en un hospital de la Provincia de Santa Elena (Ecuador), evaluándose la cantidad de antibiótico consumida entre 2018 y 2019. En el año 2018 se observó que ampicilina + sulbactam y Piperacilina + tazobactam tenían porcentajes de consumo entre 17 al $31 \%$ respectivamente, mientras que en el 2019 se evidenció un incremento de un 19\% para la Ampicilina + Sulbactam y de un 41\% para la piperacilina + tazobactam. Esta situación se debió a que el PROA ejecutado generó un desescalamiento y redujo la administración de carbapenémicos como el Meropenem e Imipenem + Cilastatina por otro tipo de antibióticos de menor toxicidad como son los inhibidores betalactámicos. Situación similar ocurrió con cefalosporinas de tercera y cuarta generación como la: ceftazidima (de uso notable ante la Pseudomonas aeruginosa y bacilos no fermentadores), la ceftriaxona y el cefepime, cuyos usos tuvieron un leve incremento dado el desescalamiento de los carbapenémicos.

Palabras clave: Resistencia Bacteriana, Programa de Optimización de Antibióticos, Selección de Antibióticos, Desescalamiento.

\footnotetext{
${ }^{1}$ Químico y farmacéutico, Docente de la Facultad de Ciencias Químicas, Universidad de Guayaquil, Ecuador. Correo electrónico: pablo.rualescar@ug.edu.ec.

${ }^{2}$ Magister en Diseño curricular, Docente de la Facultad de Ciencias Químicas, Universidad de Guayaquil, Ecuador. Correo electrónico: walter.mariscals@ug.edu.ec

${ }^{3}$ Magister en Epidemiología, Docente de la Facultad de Ciencias Químicas, Universidad de Guayaquil, Ecuador. Correo electrónico: william.jimenezj@ug.edu.ec
} 


\begin{abstract}
The implementation of Antibiotic Stewardship Programs (PROA for its Spanish acronym) seeks to increase the safety of patients through an adequate use of antibiotics through various microbiological determinations of resistance of microorganisms to the antibiotics selected for this study. In the present work, the implementation of a PROA was carried out in a Hospital of the Province of Santa Elena (Ecuador), evaluating the amount of antibiotic consumed between 2018 and 2019. In 2018 it was observed that Ampicillin + Sulbactam and Piperacillin + tazobactam had Percentages of consumption between 17 and 31\% respectively, while in 2019 there was an increase of $19 \%$ for Ampicillin + Sulbactam and $41 \%$ for Piperacillin + tazobactam. This situation was since the PROA executed generated a de-escalation and reduced the administration of carbapenems such as Meropenem and Imipenem + Cilastatin by other types of antibiotics with less toxicity such as beta-lactam inhibitors. A similar situation occurred with third and fourth generation cephalosporins such as: ceftazidime (of notable use against Pseudomonas aeruginosa and non-fermenting bacilli), ceftriaxone and cefepime, whose uses had a slight increase due to the de-escalation of carbapenems.
\end{abstract}

Keywords: Bacterial Resistance, Antibiotic Stewarship Program, Antibiotic Selection, Deescalation.

\title{
INTRODUCCIÓN
}

Desde hace muchas décadas, los microbios, en particular las bacterias, se han vuelto cada vez más resistentes a diversos antimicrobianos. El aval de la Asamblea Mundial de la Salud al Plan de Acción Mundial sobre la resistencia a los antimicrobiano, en mayo de 2015 y la Declaración política de la reunión de alto nivel de la Asamblea General sobre la resistencia, en septiembre de 2016, reconocen que la resistencia a los antimicrobianos es una amenaza para la salud pública mundial.(Organización Mundial de la Salud, 2016)

La inadecuada utilización de antimicrobianos, incluyendo su inapropiada elección, dosificación o duración, se ha identificado como una de las principales causas de selección y expansión de cepas multirresistentes. La implantación de programas de tipo "antimicrobial stewardship", más conocidos en nuestro entorno como Pro- gramas de Optimización de Antimicrobianos (PROA) conduce a un mejor uso y, con frecuencia, a una reducción en el consumo de antimicrobianos.

La finalidad de un PROA es optimizar el uso de antimicrobianos, fomentar la modificación del comportamiento relacionado con los hábitos de prescripción y dispensación, mejorar la calidad de la asistencia y los resultados de salud de los pacientes, evitar gastos innecesarios en la atención sanitaria, disminuir la aparición, la presión selectiva de bacterias resistentes, su propagación y 
prolongar la vida útil de los antimicrobianos existentes.

Por otro lado, no existen estudios regionales sobre el uso de antimicrobianos en hospitales. Existe así, una necesidad urgente de avanzar el conocimiento actual de antimicrobianos, prescripción y consumo, tanto en entornos comunitarios como hospitalarios.

\section{METODOLOGIA}

Se diseñó un estudio observacional prospectivo, desde el año 2018, fecha de implantación del PROA hasta el año de 2019.

\section{DESARROLLO}

El programa se desarrolló en la Unidad de Cuidados intensivos (UCI) de un hospital público de 6 camas, Se produjeron una media de 28 ingresos mensuales durante el año anterior a la implantación del programa. La selección de pacientes a revisar por los miembros del equipo, incluyendo una valoración inicial al ingreso y en las primeras 72 horas, así como tras 7 días de tratamiento, se realizó en base a la prescripción de los siguientes antimicrobianos: carbapenémicos, colistina, tigeciclina y Piperacilina + Tazobactam. La identificación de pacientes con tratamientos se realizó a través del programa de prescripción electrónica SAIS, mediante el farmacéutico del equipo PROA (Egea et al., 2018).

Cada una de las prescripciones fue valorada inicialmente por el equipo PROA y comentada posteriormente con el prescriptor, dando propuestas o alternativas que siguieran criterios de idoneidad, optimización, eficacia, adherencia a las guías, seguridad del paciente o coste-efectivas. El seguimiento a las $72 \mathrm{~h}$ valoró principalmente la adecuación del tratamiento al resultado microbiológico obtenido, así como el ajuste de dosis del tratamiento. Se realizó una reunión con el microbiólogo del equipo, quien aportó datos provisionales y definitivos de las muestras microbiológicas (Pan American Health Organization (PAHO)., 2018).

Para evaluar el impacto del PROA sobre la utilización de antimicrobianos, se comparó el consumo en Dosis Diarias Definidas (DDD) por 100 estancias. De acuerdo con el análisis de resistencia acumulada del área de UCI los microorganismos multirresistentes que se aislaron con mayor frecuencia fueron: enterobacterias productoras de $\beta$-lactamasas de espectro extendido (BLEE) o de carbapenemasas y las bacterias no fermentadoras multirresistentes como Acinetobacter baumannii y Pseudomonas aeruginosa (Dyar et al., 2017). 


\section{RESULTADOS}

Teniendo como punto de partida el año 2018, el cual fue el año en el que se inició la implementación del PROA en la Unidad de Cuidados Intensivos del Hospital Liborio Panchana, para la Dosis Diaria Definida (DDD) se evaluó la cantidad de antibióticos consumida en UCI entre ese periodo y el 2019 (Majumder et al., 2020).

En el año 2018 se observó que los inhibidores betalactámicos como son la Ampicilina + Sulbactam y piperacilina + tazobactam tenían porcentajes de consumo de 17 y $31 \%$ respectivamente; mientras que en el 2019 se evidencia un incremento en su uso (tabla 1). Esta situación se debe a que el Programa de Optimización de Antimicrobianos ejecutado generó un desescalamiento y redujo la administración de carbapenémicos como el Meropenem e Imipenem + Cilastatina por otro tipo de antibióticos de menor toxicidad como son los inhibidores betalactámicos: ampicilina + sulbactam y piperacilina + tazobactam (Yunquera-Romero et al., 2018).

Las bacterias detectadas en el año 2018 fueron aisladas de muestras como: orina, sangre y aspirados bronquiales. En orina se aislaron 36 cepas de Escherichia coli de diferentes pacientes y 18 cepas de Klebsiella pneumoniae, mientras que en sangre se lograron aislar 31cepas de E. coli, 28 cepas de K. pneumoniae y 11 cepas de Pseudomonas aeruginosa de diversos individuos (Egea et al., 2018).

El aislamiento de $P$. aeruginosa en orina no fue significativo motivo por el cual no se incluyó en el porcentaje de análisis acumulado de la resistencia antimicrobiana en UCI del período 2018 (tabla 2). Según se evidencia en el cuadro la E. coli en orina ante la ampicilina presentó un $89 \%$ de resistencia, motivo por el cual no sería indicada para un tratamiento ante este microorganismo. La piperacilina + tazobactam en cambio con un $33 \%$ de resistencia se muestra activa en contra de la $E$. coli (Yau et al., 2021).

Pese a que la $E$. coli es sensible ante el ertapenem, meropenem, e imipenem, dado a que la piperacilina + tazobactam presenta menores efectos secundarios que los carbapenémicos, se prefiere el uso de la piperacilina+ tazobactam (Carmona et al., 2018).

Respecto a los microrganismos multirresistentes acorde a sus mecanismos de resistencia se observa en la (tabla 3) que la mayor cantidad de bacterias aisladas en UCI correspondieron a $E$. coli, $K$. pneumoniae y A. baumanni (Logan \& Weinstein, 2017). 
Escherichia y Klebsiella presentan diferentes tipos de mecanismos de resistencia encontrándose en UCI a los productores de BLEE (betalactamasas de espectro extendido), productores de carbapenemasas (enzimas que generan resistencia a los carbapenémicos), multirresistentes (resistencia a tres o más familias de antimicrobianos), en Pseudomonas resistentes productoras de BLEE y Acinetobacter sin carbapenemasas con otros tipos de multirresistencia no carbapenemasas (Salavert et al., 2018).

Un 44\% de mecanismos de resistencia de E. coli productora de BLEE, se encontró durante el 2018 y un descenso posterior al 41\% en el 2019. Para K. pneumoniae Productor de BLEE durante el 2018 se evidenció un porcentaje de mecanismo de resistencia del 52\% y un descenso al 2019 del 47\%. Para K. pneumoniae productor de carbapenemasa hay una reducción del $1 \%$ entre el 2018 y 2019. $P$. aeruginosa multirresistentes en cambio presentaron un decrecimiento del 5\% (de 14 a 9\%) y para $P$. aeruginosa productor de BLEE y A. baumanni multirresistente se evidenció un decrecimiento de un 1 y $8 \%$ respectivamente(Villegas \& Zurita, 2016).

El porcentaje de Desescalada nos muestra que, de los antibióticos administrados en la UCI del hospital durante el periodo propuesto, fueron los carbapenémicos los que tuvieron un mayor descenso en su utilización 10\% (tabla 4). Esto a raíz de que el Programa de Optimización de Antimicrobianos (PROA) que se ejecutó en esta institución, propició el uso de inhibidores de betalactámicos como son la ampicilina y sulbactam junto con la piperacilina y tazobactam en vez de los carbapenémicos (ertapenem, meropenem e imipenem) (Delcour, 2009).

\section{CONCLUSIONES}

Los programas de optimización de antibióticos buscan contrarrestar la resistencia bacteriana a través de un uso adecuado de estos medicamentos para proporcionar mejores resultados clínicos, un tratamiento rentable y, reducir o estabilizar la resistencia bacteriana a nivel clínico y hospitalario.

\section{REFERENCIAS}

Alcalá-Hernández, C. F., de la Rosa, L. A., Pedraza-Chaverri, J., Medina-Campos, O. N., Meza Ireta, S. A., Wall-Medrano, A., . . Álvarez-Parrilla, E. (2017). Caracterización fitoquímica de un extracto de Capsicum annuum cv. "Jalapeño" y su actividad citotóxica en células 3T3L1. Revista Mexicana de Ciencias Farmacéuticas, 48(3), 10.

Alexakis, P., \& Siriopoulos, C. (1999). The international stock market crisis of 1997 and the dynamic relationships between asian stock markets: Linear and nonlinear Granger causality tests. Managerial Finance(25), 22-38.

AMA. (2013). American Marketing Association. Obtenido de Journal of Marketing Reseach: https://www.ama.org/publications/JournalOfMarketing/Pages/Current-Issue.aspx

Andreasen, A. (1994). Social Marketing: Its definition and Domain. Journal of Public Policy and Marketing, 13(Spring), 108-114. 
Arcotel. (2018). Estadísticas Anuales Líneas Móviles. Recuperado el 26 de Enero de 2018, de Agencia de Regulación y Control de Telecomunicaciones: http://www.arcotel.gob.ec/servicio-movil-avanzado-sma/

Barboza, A. (2016). Sobre el Método de la interpretación documental y el uso de las imágines en la sociología. Sociedade e Estado, 21(2), 391-414.

Blodgett, J., Lu, L.-C., \& Rose, G. (2001). Ethical Sensitivity to Stakeholder Interest: A CrossCultrural Comparison. Journal of Academy of Marketing Science, 29(2), 190-202.

Castillo, O. R. (2020). DETERMINACIÓN DE POLIFENOLES TOTALES Y ACTIVIDAD ANTIOXIDANTE DEL EXTRACTO METANÓLICO DE TRES VARIEDADES DE PIMIENTOS (CAPSICUM ANUUM. 78.

Chiarelli, J., Marconi, M., Pistani, M., Waingarten, S., \& Knopoff, E. (Junio de 2017). Sistema de farmacovigilancia: conocimiento y actitudes de los médicos del primer nivel de atención y tasa de notificación de efectos adversos para medicación antituberculosis. Revista Americana de Medicina Respiratoria, 17(2).

Claro. (Enero de 2018). Claro. Recuperado el 2 de Febrero de 2018, de Quienes Somos: https://www.claro.com.ec/personas/institucional/quienes-somos/

CNT-EP. (2018). La Corporación Nacional de Telecomunicaciones CNT EP. Recuperado el 2 de Febrero de 2018, de CNT: http://corporativo.cnt.gob.ec/historia-de-las-telecomunicacionesen-el-ecuador/

Consejo General de Colegios Oficiales de Farmacéuticos. (2010). El papel del farmacéutico en la seguridad del paciente. Recuperado el 29 de Marzo de 2021, de El papel del farmacéutico en la seguridad del paciente:

https://www.portalfarma.com/Profesionales/campanaspf/categorias/Documents/Documentos -Publica/2010_Informe_Tecnico_Seguridad_del_paciente.pdf

Coralí, P. O., \& Yomira, P. B. (2019). "Influencia del estado de madurez en el índice de carotenoides del pimiento morrón (Capsicum annuum), utilizando visión artificial”. LAMBAYEQUE - PERÚ.

Cruz, M., Ruiz, A., Furones, J., \& Palenzuela, I. (2015). Conocimientos sobre farmacovigilancia del personal de estomatología en municipios seleccionados. Revista de Ciencias Médicas La Habana, 21(3).

Drucker, P. (1984). The new meaning of corporate social responsi-bility. California Management Review, $X X V I(2), 53-63$.

García Carlos, L. M. (2016). Determinación de Vitamina C en pimiento Capsicum annuum por voltametría de barrido linel. Revista Talentos.

Garcia, A. R. (2017). Efecto del Tratamiento Termico en Horno por aire Forzado sobre el contenido de Vitamina C y Provitamina A del pimiento Morron Amarillo (Capsicum ANNUUM L.).

Gómez, P. (2003). La gestión de marketing de ciudades y áreas metropolitanas: de la orientación al producto a la orientación al marketing. Cuadernos de Gestión, 3(1), 11-25.

Gonzále, C. G., Bonilla, H. R., \& Calderón, B. L. (Abril de 2017). Cuantificación de ácido ascórbico en pimientos comercializados en la zona 7 del Ecuador aplicando voltamperometría. Cumbres, Revista Cientifica, 3(2).

Gordhon, Y., \& Pedayachee, N. (2020). Evaluating the knowledge, attitudes and practices of healthcare workers towards adverse drug reaction reporting at a public tertiary hospital in Johannesburg. International Journal of Africa Nursing Sciences, 12.

Green Paper. (2011). Green Paper on corporate social responsibility. Recuperado el 2018 de Enero de 2018, de Europa: http://europa.eu/legislation

Hunt, S. (1983). General theories and the fundamentalexplananda of marketing. Journal of Marketing, 47(4), 9-17.

Jiménez, G., Debesa, F., González, B., Ávila, J., \& Pérez, J. (2006). El Sistema Cubano de Farmacovigilancia, seis años de experiencia en la detección de efectos adversos. Rev Cubana Farm, 40(1), 1-8. Obtenido de http://scielo.sld.cu/pdf/far/v40n1/far02106.pdf Jiménez, G., García, A., Gálvez, A., Alfonso, I., Lara, M., \& Calvo, D. (2014). Medicamentos 
notificados como productores de reacciones adversas graves en Cuba en un período de diez años. Revista Cubana de Salud Pública, 40(4), 263-275. Obtenido de https://www.medigraphic.com/pdfs/revcubsalpub/csp-2014/csp144c.pdf

Kotler, P., \& Levy, S. (1969). Broading the concept of Marketing. Journal of Marketing, 33(January), 10-15.

Kotler, P., \& Zaltman, G. (1971). Social marketing: An approach toplanned social change. Journal of Marketing, 35, 3-12.

Lideres. (Enero de 2014). Revista Lideres. Recuperado el 12 de Enero de 2018, de En 20 años, la telefonía móvil superó las expectativas: http://www.revistalideres.ec/lideres/20-anostelefonia-movil-supero.html.

Maignan, I., \& Ferrell, O. (2004). Corporate social responsibility and marketing: an integrative framework. Journal of the Academy of Marketing Science, 32(1), 19-23.

Mata, J., Ortiz, M., Islas, H., Diaz, M., De León, V., \& Tenorio, L. (Octubre-diciembre de 2017). Impacto de una intervención educativa en los conocimientos en farmacovigilancia y en el reporte de reacciones adversas a los medicamentos de profesionales de la salud en un hospital público de segundo nivel de atención en el Estado de México, México. Revista Mexicana de Ciencias Farmacéuticas, 48(4).

Maza, J., Aguilar, L., \& Mendoza, J. (2018). Farmacovigilancia: un paso importante en la seguridad del paciente. Medigraphic, 72(1), 47-53.

Miron, D., Petcu, M., \& Sobolevschi, M. (2011). Corporate Social Responsibility and sustainable competitiveness. Amfiteatru Economic Journal, A.S.E.(29), 163-180. Muberra, G., \& Perihan, E. (Enero de 2019). Healthcare professionals pharmacovigilance knowledge and adverse drug reaction reporting behavior and factors determining the reporting rates. Journal of Drug Assessment, 8(1).

Nerd, N. (2018). Determination of Vitamin C in Various Colours of Bell Pepper. Department of Pharmacy, 14. Obtenido de https://pdfs.semanticscholar.org/3c78/bbb27a445521302bd26c370463f9b8393c63.pdf

O, A. C., Jennifer Y, P. B., \& R, N. L. (Octubre de 2019). Índice de carotenoides de pimiento morrón (Capsicum annuum) basado en la medición de color, utilizando imágenes hiperespectrales y digitales. Scielo, 10(4).

Pablo Preciado-Rangel, A. A.-S.-C.-P.-H.-P.-H. (2019). INFLUENCIA DEL POTASIO EN EL CONTENIDO NUTRACÉUTICO Y ANTIOXIDANTE DEL PIMIENTA SERRANO (Capsicum annuum L.). 53(4), 581-591.

Patricio Yánez, D. B. (2015). Características morfológicas y de concentración de capsaicina en cinco especies nativas del género Capsicum cultivadas en Ecuado. LA GRANJA: Revista de Ciencias de la Vida, 21.

Ramos, J., \& Periáñez, I. (2003). Delimitación del Marketing con Causa o Marketing Social Corporativo mediante el análisis de empresas que realizan acciones de responsabilidad social. Cuadernos de Gestión, 3(1), 65-82.

Romero, K. (Abril-Junio de 2018). El conocimiento de la Farmacología en el profesional de enfermería. Enfermería Investiga, Investigación, Vinculación, Docencia y Gestión, 3(2).

Ruiz, A., García, A., Jiménez, G., Alfonso, I., Pérez, B., \& Carrazana, A. (2013). Farmacovigilancia de fitofármacos y apifármacos en Cuba durante 2006-2010. Revista Cubana de Plantas Medicinales, 18(2), 173-186. Obtenido de http://scielo.sld.cu/pdf/pla/v18n2/pla02213.pdf

Sáez, M., Sánchez, N., Jiménez, S., Alonso, N., \& Valverde, J. (Marzo-Abril de 2016). Tratamiento del dolor en el anciano: opioides y adyuvantes. Revista de la Sociedad Española del Dolor, 23(2).

Telefónica S.A. (2018). Movistar. Recuperado el 3 de Febrero de 2018, de Historia de Movistar: https://www.movistar.com.ec/

Torres, B. (2006). Metodología de la Investigación. Mexico D.F: Pearson.

Trabanca, Y., Jiménez, G., Alfonso, i., Pavón, K., \& Albear, F. (Marzo-Abril de 2018). Implementación del Programa de Notificación de Efectos Adversos por Pacientes en Guantánamo. Revista de Información Científica, 97(2).

Vaaland, T., Heide, M., \& Grønhaug, K. (2008). Corporate social responsibility: investigating 
theory and research in the marketing context. European Journal of Marketing, (9/10), 927953.

Vázquez, M. (2011). Investigación en educación matemática: objetivos, cambios, criterios, método y difusión. Education Siglo XXI, 29(2), 173-198.

Vicente, M. Azucena; Mediano, Lucía; . (2002). Propuestas para una segmentación estragégica del mercado ecológico . Cuadernos de Gestión, 2(1).

WBCSD. (2012). World Business Council for Sustainable Development, . Recuperado el 15 de Enero de 2018, de . Corporate social responsibility: Meeting changing expectations [pdf].: http://www.wbcsd.org 